\title{
DÜBLIN
}

Technological University Dublin

ARROW@TU Dublin

2011-05-01

\section{Humidity Sensor Based on a Single-mode Hetero-core Fiber Structure}

\author{
Qiang Wu \\ Technological University Dublin, qiang.wu@tudublin.ie \\ Yuliya Semenova \\ Technological University Dublin, yuliya.semenova@tudublin.ie \\ Jinesh Mathew \\ Technological University Dublin
}

See next page for additional authors

Follow this and additional works at: https://arrow.tudublin.ie/engscheceart

Part of the Electrical and Computer Engineering Commons

\section{Recommended Citation}

Wu, Q. et al. (2011) Humidity sensor based on a single-mode hetero-core fiber structure. Optics Letters, Vol. 36, no. 10, pp. 1752-1754. doi:10.1364/OL.36.001752

This Article is brought to you for free and open access by the School of Electrical and Electronic Engineering at ARROW@TU Dublin. It has been accepted for inclusion in Articles by an authorized administrator of ARROW@TU Dublin. For more information, please contact arrow.admin@tudublin.ie, aisling.coyne@tudublin.ie, gerard.connolly@tudublin.ie.

Funder: Science Foundation Ireland under grant 07/SK/ 11200, the Irish Research Council for Science, Engineering and Technology, and Marie-Curie Actions under FP7

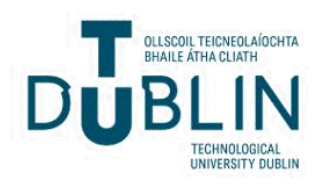


Authors

Qiang Wu, Yuliya Semenova, Jinesh Mathew, Pengfei Wang, and Gerald Farrell

This article is available at ARROW@TU Dublin: https://arrow.tudublin.ie/engscheceart/101 


\title{
Humidity sensor based on a single-mode hetero-core fiber structure
}

\author{
Qiang Wu,* Yuliya Semenova, Jinesh Mathew, Pengfei Wang, and Gerald Farrell \\ Photonics Research Center, School of Electronic and Communications Engineering, \\ Dublin Institute of Technology, Kevin Street, Dublin 8, Ireland \\ ${ }^{*}$ Corresponding author: qiang.wu@dit.ie
}

Received February 9, 2011; revised March 23, 2011; accepted April 5, 2011; posted April 6, 2011 (Doc. ID 142499); published May 4, 2011

\begin{abstract}
Using a small-core single-mode fiber (SCSMF), a novel relative humidity (RH) sensor based on an SMF28-SCSMFSMF28 fiber structure was proposed in this paper. By depositing a humidity sensitive material, such as poly (ethylene oxide) (PEO) on the bare SCSMF fiber, the proposed structure can act as an RH sensor with high sensitivity. Experiments demonstrated that the proposed RH sensor with PEO coating can achieve a sensitivity of $430 \mathrm{~nm}$ per relative humidity unit (RHU) in the RH range from $80 \%$ to $83 \% \mathrm{RH}$ and a sensitivity of $50 \mathrm{~nm}$ per RHU in the RH range from $83 \%$ to $95 \%$ RH. (c) 2011 Optical Society of America
\end{abstract}

OCIS codes: $\quad 060.2370,060.2340$.

Monitoring and measuring relative humidity is very important in a range of areas, such as food processing, chemical manufacturing, civil engineering, weather observations, and air-conditioning control. Traditional electronic RH sensors are based on monitoring the changes in electrical conductivity or capacitance and, hence, have the disadvantage of inaccuracy due to electrical leakage, especially in a high humidity environment. Compared with electronic RH sensors, optical fiber based RH sensors offer numerous advantages, such as immunity to electromagnetic interference, small size, low weight, and remote monitoring. A wide range of $\mathrm{RH}$ sensing techniques based on optical fibers has been reported previously, including long period gratings (LPG) [1-3], fiber Bragg gratings [4,5], side polished fibers [6], plastic

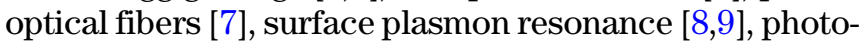
nic crystal fiber interferometer [10], and tapered optical fibers $[11,12]$. Recently Akita proposed to use a heterocore optical fiber as a humidity sensor, which is based on a multimode-singlemode-multimode fiber structure with the benefits of simplicity and low cost [13].

In our previous research, we have demonstrated that a single-mode-multimode-single-mode (SMS) fiber structure can act as both a temperature and displacement sensor $[14,15]$. If the cladding of a multimode fiber is etched away, such an SMS fiber structure can be used as a refractive index (RI) sensor $[16,17]$. However, etching of the fiber cladding is often complex and dangerous due to the chemicals used in the etching process. More importantly, the control of the etching process to achieve a repeatable fiber diameter is very difficult and the surface of the etched fiber is normally rough, which influences the transmission behavior of the etched fiber. In this paper, we propose to use a single-mode optical fiber with a very small core diameter instead of the multimode fiber in the SMS structure. By depositing a thin layer humidity-sensitive material, such as PEO on the surface of the fiber, the proposed structure can act as an RH sensor with high sensitivity.

The proposed RH sensor structure is shown in Fig. 1.

A conventional SMF28 fiber is used as the attaching fibers to the SCSMF. Both the SMF28 and SCSMF fibers in Fig. 1 have a step index profile. The SCSMF effectively has four layers, if one includes the air surrounding the PEO layer. Since the core diameter of the SMF28 is much larger than that of the SCSMF, the light injected into the SCSMF from SMF28 will excite multiple modes propagating within the cladding of the SCSMF. When the RI of PEO $\left(n_{3}\right)$ is less than that of the cladding $\left(n_{2}\right)$, there are multiple eigenmodes transmitted within the SCSMF. Assuming the field profile within the SCSMF as $\Psi_{m}(r)$, the input field at the SCSMF can be written as

$$
E(r, 0)=\sum_{m=1}^{M} b_{m} \Psi_{m}(r),
$$

where $E(r, 0)$ is the eigenmode of the SMF28, $\Psi_{m}(r)$ is $m$ th eigenmode of the step index optical fiber SCSMF, which was given by Tsao [18], and where $b_{m}$ is the excitation coefficient for each mode, which can be expressed as

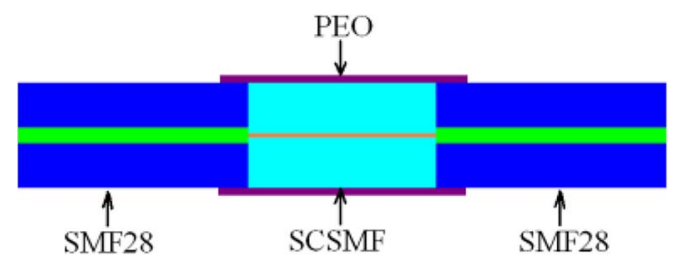

(a)

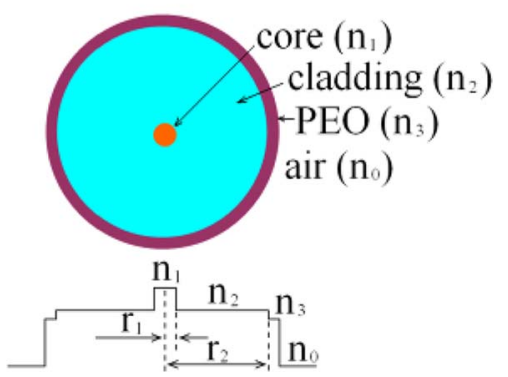

(b)

Fig. 1. SMF28-SCSMF-SMF28 fiber structure based humidity sensor. (a) View along fiber axis and (b) cross-section view of the SCSMF section. 


$$
b_{m}=\frac{\int_{0}^{\infty} E(r, 0) \Psi_{m}(r) r d r}{\int_{0}^{\infty} \Psi_{m}(r) \Psi_{m}(r) r d r}
$$

The field within the SCSMF section at a propagation distance $z$ can thus be calculated by

$$
E(r, z)=\sum_{m=1}^{M} b_{m} \Psi_{m}(r) \exp \left(j \beta_{m} z\right),
$$

where $\beta_{m}$ is the propagation constant of each eigenmode within the SCSMF. The transmitted power to the output SMF28 thus can be expressed as

$$
L_{s}(\boldsymbol{z})=10 \cdot \log _{10}\left(\frac{\left|\int_{0}^{\infty} E(r, z) E(r, 0) r d r\right|^{2}}{\int_{0}^{\infty}|E(r, z)|^{2} r d r \int_{0}^{\infty}|E(r, 0)|^{2} r d r}\right) .
$$

Simulations based on the above formula were carried out. Figure 2 gives wavelength shift versus RI of the overlay at two different overlay thicknesses. The parameters used in this simulation are $n_{1}=1.451, n_{2}=1.445, n_{4}=1$, $r_{1}=1.15 \mu \mathrm{m}, r_{2}=62.5 \mu \mathrm{m}$.

Figure 2 shows that as the RI of the overlay increases, the wavelength shift increases and the structure with a thicker overlay has a larger wavelength shift compared to that with a thinner overlay. For an RH sensor based on this structure, as the $\mathrm{RH}$ of the air increases, the RI of the PEO decreases, hence, the wavelength decreases. By monitoring the spectral response of the sensor, the $\mathrm{RH}$ can be determined, if a suitable calibration has taken place.

Experiments based on the above structure were carried out. The SMF28 fiber is a conventional fiber used in optical communications and supplied by Corning. The SCSMF fiber has core and cladding refractive indices of 1.467 and 1.461, respectively, at $532 \mathrm{~nm}$ with mode field diameter of $3.5 \pm 0.5 \mu \mathrm{m}$ at $515 \mathrm{~nm}$. The SMF28SCSMF-SMF28 fiber structure was fabricated by fusion splicing. The coating of the SCSMF was fully stripped and the length of the SCSMF section was $40 \mathrm{~mm}$, which was carefully selected to achieve a relatively narrow bandwidth and a compact sensor length. The humidity sensing material used in this experiment is PEO, which

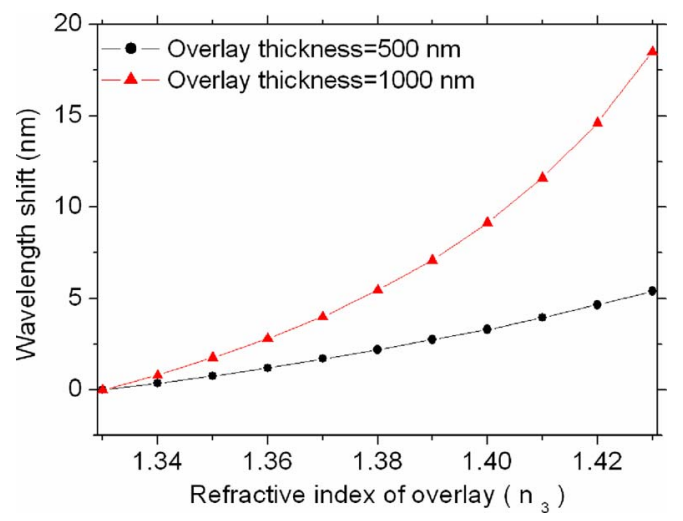

Fig. 2. Simulated wavelength shift versus RI of the overlay. has an RI of 1.4539 at the wavelength of $589.3 \mathrm{~nm}$. The PEO solution containing $10 \mathrm{wt}$ \% was prepared by mixing PEO and deionized water. The PEO was then deposited by passing the SCSMF section through a drop of the PEO solution using a motor controlled translation stage. By changing the translation speed, coatings with different thicknesses can be achieved. In our experiments two samples were prepared with two different translation speeds: $4 \mathrm{~mm} / \mathrm{s}$ (marked as sensor 1) and $10 \mathrm{~mm} / \mathrm{s}$ (marked as sensor 2). It should be noted that due to limitations of the experimental equipment, the thickness of the coating could not be exactly measured. However, under observation using a microscope with $600 \times$ magnifications, it is concluded that the coating thickness for both samples is less than $1 \mu \mathrm{m}$ and that the coating of sensor two is seen to be thicker than that of sensor one. Optimal coating thickness could be achieved by dynamic monitoring of spectral response of the SMF28SCSMF-SMF28 fiber structure.

The sensors were then placed in a climate control chamber. Light from a broadband optical source was injected into the sensor and the output signal was monitored using an optical spectrum analyzer (OSA). The humidity within the chamber was first increased to $95 \% \mathrm{RH}$, and then was decreased gradually to $40 \%$ $\mathrm{RH}$ with steps of $0.1 \% \mathrm{RH}$ (maximum available resolution of the setup) at a fixed temperature of $19.2 \pm 0.5^{\circ} \mathrm{C}$. The spectral responses of the two sensors at different $\mathrm{RH}$ values are shown in Fig. 3 .

Figure 3 shows that as the RH increases, the central wavelength of the spectral response increases for both sensors. This is due to the fact that as $\mathrm{RH}$ decreases, the RI of the PEO increases, and, hence, the eigenmodes excited in the SCSMF section change resulting in the changes to the light coupled to the output SMF28. Figure 3 also shows that as the RH decreases, the bandwidth of the spectra increases and the spectra shape becomes irregular, especially for sensor two. This could possibly be explained as water vapor diffusion inside the PEO introducing complex changes to the polymer material, such as optical density, RI, and surface morphology [2].

For sensor one the range of the central wavelength shift was more than $20 \mathrm{~nm}$ when the $\mathrm{RH}$ decreased from $95 \% \mathrm{RH}$ to $40 \% \mathrm{RH}$, while for sensor two this value was almost two times larger. This indicates that sensor two

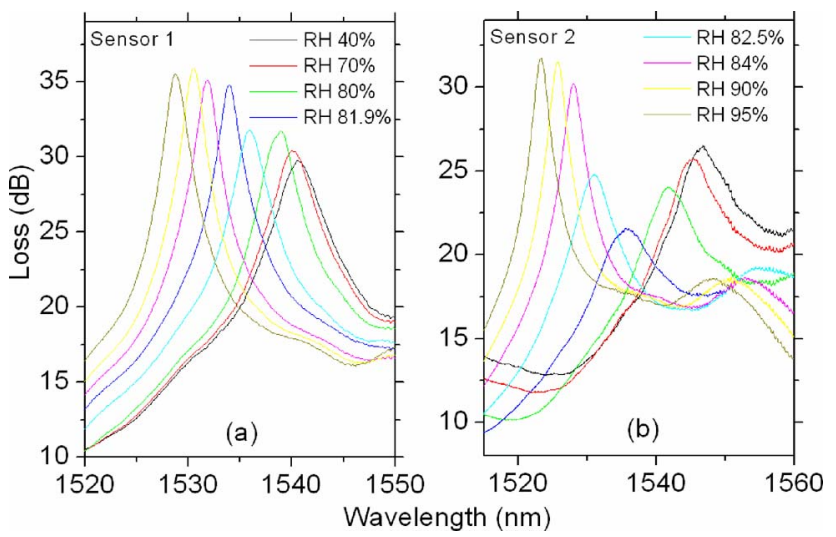

Fig. 3. Spectral responses of (a) sensor one and (b) sensor two at different $\mathrm{RH}$ values. 


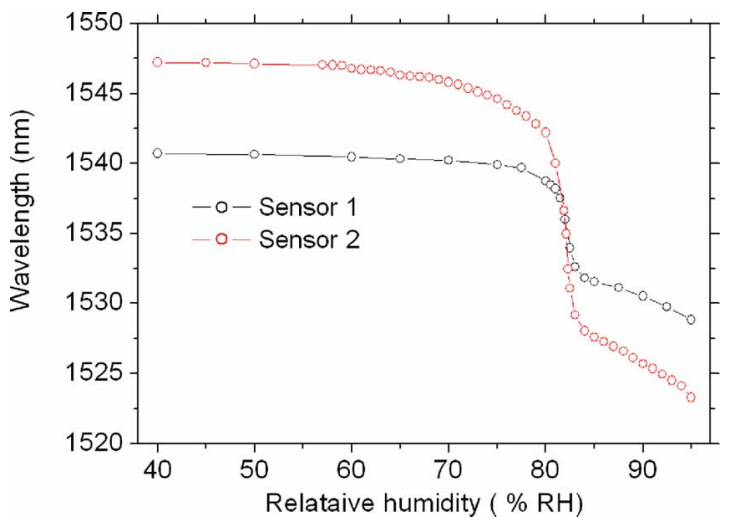

Fig. 4. Resonant wavelength shift of the SMF28-SCSMFSMF28 fiber structure based $\mathrm{RH}$ sensor versus different $\mathrm{RH}$ values.

has a higher sensitivity than sensor one. Figure 4 shows the measured central wavelength variations versu $\mathrm{RH}$. It is noted that in this study, the central wavelength is the $3 \mathrm{~dB}$ mean wavelength measured by the OSA, which is a more reliable measurement compared to the peak central wavelength.

Figure 4 shows that for both sensors, as $\mathrm{RH}$ increases from $40 \% \mathrm{RH}$ to $95 \% \mathrm{RH}$, the central wavelength of the response shifts to a shorter wavelength. For sensor one, there is no significant wavelength shift in the range of $\mathrm{RH}$ from $40 \%$ to $70 \% \mathrm{RH}$; when the $\mathrm{RH}$ is higher than $70 \%$, the wavelength shift is significant, especially in the range from $80 \%$ to $83 \% \mathrm{RH}$ where it is equal to $6 \mathrm{~nm}$. In the $\mathrm{RH}$ range from $83 \% \mathrm{RH}$ to $95 \% \mathrm{RH}$, there is a circa $4 \mathrm{~nm}$ wavelength shift. The total wavelength shift in the $\mathrm{RH}$ range from $40 \% \mathrm{RH}$ to $95 \% \mathrm{RH}$ for sensor one is circa $12 \mathrm{~nm}$. Sensor two shows a similar wavelength shift behavior but with a larger wavelength shift value $(24 \mathrm{~nm})$ in the $\mathrm{RH}$ range from $40 \%$ to $95 \% \mathrm{RH}$, indicating that sensor two has higher sensitivity than sensor one, from which it is possible to conclude that the thickness of the PEO layer has an effect on the sensor sensitivity. Assuming the OSA has a wavelength resolution of $0.01 \mathrm{~nm}$, the proposed sensors have humidity resolutions of $0.005 \% \mathrm{RH}$ and $0.0023 \% \mathrm{RH}$ in the $\mathrm{RH}$ range from $80 \% \mathrm{RH}$ to $83 \%$ $\mathrm{RH}$, and resolutions of $0.03 \% \mathrm{RH}$ and $0.02 \% \mathrm{RH}$ in the $\mathrm{RH}$ range from $83 \%$ to $95 \% \mathrm{RH}$ for sensor one and two, respectively. By comparison with an LPG humidity sensor coated with a similar humidity sensing material of $\mathrm{PEO} / \mathrm{CoCl}_{2}$, which has a maximum wavelength shift of $7.5 \mathrm{~nm}$ at $\mathrm{RH}$ range from $77 \%$ to $95 \%$, the sensor two used in our experiment has a higher sensitivity with a wavelength shift of $20.5 \mathrm{~nm}$ in the same $\mathrm{RH}$ range.

In conclusion, we have proposed an RH sensor based on an SMF28-SCSMF-SMF28 fiber structure. Compared to the existing optical fiber based $\mathrm{RH}$ sensors, the $\mathrm{RH}$ sensor proposed in this paper has the advantages of high resolution, low cost, and ease of fabrication.

It should be noted that in this paper we have carried out experiments for $\mathrm{RH}$ values less than $95 \% \mathrm{RH}$ due to the fact that PEO dissolves if the humidity is higher than $95 \% \mathrm{RH}$, which could result in damage to the sensor. However, if a different humidity-sensitive material is used as a coating, for example, hydrogel [1], which is stable in a high humidity range up to $100 \%$, then this SMF28-SCSMF-SMF28 fiber structure based RH sensor can be used to measure $\mathrm{RH}$ values up to $100 \% \mathrm{RH}$.

This work was supported by Science Foundation Ireland under grant 07/SK/I1200, the Irish Research Council for Science, Engineering and Technology, and Marie-Curie Actions under FP7.

\section{References}

1. Y. Liu, L. W. Wang, M. Zhang, D. S. Tu, X. H. Mao, and Y. B. Liao, IEEE Photon. Technol. Lett. 19, 880 (2007).

2. M. Konstantaki, S. Pissadakis, S. Pispas, N. Madamopoulos, and N. A. Vainos, Appl. Opt. 45, 4567 (2006).

3. I. Del Villar, C. R. Zamarreno, M. Hernaez, F. J. Arregui, and I. R. Matias, Opt. Express 18, 20183 (2010).

4. C. Zhang, W. Zhang, D. J. Webb, and G. D. Peng, Electron. Lett. 46, 643 (2010).

5. Y. P. Miao, B. Liu, H. Zhang, Y. Li, H. B. Zhou, H. Sun, W. H. Zhang, and Q. D. Zhao, IEEE Photon. Technol. Lett. 21, 441 (2009).

6. A. Alvarez-Herrero, H. Guerrero, and D. Levy, IEEE Sens. J. 4, 52 (2004).

7. S. Muto, O. Suzuki, T. Amano, and M. Morisawa, Meas. Sci. Technol. 14, 746 (2003).

8. M. N. Weiss, R. Srivastava, and H. Groger, Electron. Lett. 32, 842 (1996).

9. Z. M. Qi, I. Honma, and H. S. Zhou, Opt. Lett. 31, 1854 (2006).

10. J. Mathew, Y. Semenova, G. Rajan, and G. Farrell, Electron. Lett. 46, 1341 (2010).

11. C. Bariain, I. R. Matias, F. J. Arregui, and M. Lopez-Amo, Sens. Actuators B Chem 69, 127 (2000).

12. X. F. Huang, D. R. Sheng, K. F. Cen, and H. Zhou, Sens. Actuators B Chem 127, 518 (2007).

13. S. Akita, H. Sasaki, K. Watanabe, and A. Seki, Sens. Actuators B Chem 147, 385 (2010).

14. Q. Wu, A. M. Hatta, P. Wang, Y. Semenova, and G. Farrell, IEEE Photon. Technol. Lett. 23, 130 (2011).

15. Q. Wu, A. M. Hatta, Y. Semenova, and G. Farrell, Appl. Opt. 48, 5451 (2009).

16. Q. Wang and G. Farrell, Opt. Lett. 31, 317 (2006).

17. Q. Wu, Y. Semenova, P. Wang, and G. Farrell, Opt. Express 19, 7937 (2011).

18. C. Tsao, Optical Fibre Waveguide Analysis (Oxford, 1992). 